\title{
Evaluación de la toxicidad de metales pesados en dos suelos agrícolas de Colombia mediante bioensayos
}

\section{Evaluation of heavy metal toxicity in two Colombian agricultural soils using}

\author{
Luis E. Cortés ${ }^{1 *}$, Francisco J. Martin², Margarita M. Sarria ${ }^{3}$ \\ Recibido para publicación: Julio 24 de 2016 - Aceptado para publicación: Mayo 18 de 2017
}

\begin{abstract}
RESUMEN
La presente investigación tuvo como objetivo determinar la toxicidad de $\mathrm{Cu}, \mathrm{Cd}, \mathrm{Ni}, \mathrm{Pb}$ y $\mathrm{Zn}$, en dos suelos agrícolas colombianos, Andisol y Vertisol, mediante bioensayos con Lactuca sativa y Vibrio fischeri. Los metales se adicionaron individualmente a los suelos hasta superar el nivel de intervención de Andalucía. Posteriormente se incubaron a $25^{\circ} \mathrm{C}$ y $60 \%$ de humedad, durante 60 y 90 días, respectivamente. El diseño experimental fue un completamente al azar con tres repeticiones. Luego de cada incubación se determinó la concentración de los metales en extractos acuosos, y con ellos se efectuaron bioensayos con Lactuca Sativa y Vibrio fischeri. Se efectuó análisis estadístico de resultados, comparaciones post hoc (Tukey), correlaciones y análisis de componentes principales, para encontrar los factores que afectaron los organismos en estudio. Se encontró que el Vertisol presentó mayor capacidad de inmovilización de los metales, en función de su contenido de arcillas y $\mathrm{pH}$ neutro, lo cual se reflejó en menor toxicidad sobre las especies en ensayo, indicando que este suelo actuó como protectante contra la movilidad de contaminantes en solución del suelo. El ensayo con $V$. fischeri presentó mayor sensibilidad a los contaminantes, permitiendo una mejor discriminación de los niveles de toxicidad. La capacidad de ambos suelos de inhibir la toxicidad se incrementó con el tiempo, posiblemente por interacción de los metales con los diferentes componentes de los suelos, y la disminución de su movilidad por mecanismos como la formación de complejos estables, y se relacionó con el incremento del $\mathrm{pH}$.
\end{abstract}

Palabras clave: Andisol, Contaminación, Lactuca sativa, Vertisol, Vibrio fischeri.

\begin{abstract}
This study aimed to determine the toxicity of $\mathrm{Cu}, \mathrm{Cd}$, $\mathrm{Ni}, \mathrm{Pb}$ and $\mathrm{Zn}$ in two Colombian agricultural soils, Andisol and Vertisol, by bioassays with Lactuca Sativa and Vibrio fischeri. The metals were individually added to soils exceeding the intervention level of Andalusia. Subsequently, they were incubated at $25{ }^{\circ} \mathrm{C}$ and $60 \%$ humidity for 60 and 90 days, respectively. The experimental design was completely randomized with three replicates. After each incubation, the concentration of the metals in aqueous extracts was determined, and bioassays were carried out with Lactuca Sativa and Vibrio fischeri. Statistical analysis of results, post hoc comparisons (Tukey), correlations and principal component analysis were performed to find the factors that affected the organisms under study. It was found that Vertisol showed a higher immobilization capacity of all metals, based on their clay content and neutral $\mathrm{pH}$, which was reflected in less toxicity on the organisms tested, indicating that this soil acted as a protection against mobility of pollutants in soil solution. The V. fischeri test presented greater sensitivity to contaminants, allowing a better discrimination of toxicity levels. The ability of both soils to inhibit toxicity increased over time, possibly through the interaction of metals with the different soil components, and the decrease in their mobility by mechanisms such as the formation of stable complexes, and was related to the increase of $\mathrm{pH}$.
\end{abstract}

Key words: Andisol, Contamination, Lactuca sativa, Vertisol, Vibrio fischeri.

\footnotetext{
1* Ph.D. Profesor Asociado. Departamento de Ciencias Básicas, Facultad de Ingeniería y Administración, Universidad Nacional de Colombia, Palmira, Colombia. Carrera 32 No 12-00 Barrio Chapinero. Teléfono 2868888 Ext: 34428. Email. lecortesp@unal.edu.co autor para correspondencia.

2 Ph.D. Profesor titular. Departamento de Edafología y Química Agrícola, Universidad de Granada. Granada, España.

3 M.Sc. Facultad de Ciencias Agropecuarias, Universidad Nacional de Colombia, Palmira, Colombia.
} 


\section{INTRODUCCIÓN}

En las últimas décadas, a nivel mundial, el contenido de metales pesados en suelos agrícolas se ha incrementado (Huang et al. 2015). Su acumulación se debe a la intemperización del material parental y principalmente por actividades antropogénicas como la deposición de residuos industriales y domésticos, relaves mineros, lodos de depuradora, aplicación de estiércol de ganado y aves, fertilizantes inorgánicos, cal, agroquímicos, agua de riego y pesticidas (Wang et al. 2015). Esto puede ocasionar un deterioro en las aguas subterráneas, en la calidad de los cultivos y a través de la cadena alimenticia, una potencial afectación en la salud humana de tipo cancerígeno, teratogénicos, genotóxicos y/o mutagénicos (Marrugo-Negrete et al. 2017; Hu et al. 2007; Cortés et al. 2015).

Algunos elementos son considerados esenciales como $\mathrm{Co}, \mathrm{Cu}, \mathrm{Fe}, \mathrm{Mn}, \mathrm{Mo}, \mathrm{Ni}$ y $\mathrm{Zn}$, indispensables para el óptimo funcionamiento y reproducción de microorganismos, plantas, animales, incluido el hombre (Sherameti y Varma 2010; Kabata-Pendias 2011). No obstante, al igual que los demás elementos que componen el grupo de metales pesados, a altas concentraciones pueden ser tóxicos para los organismos (Bolan et al. 2014).

Para evaluar el riesgo ecológico que puede ocurrir en suelos contaminados por metales pesados se realizan bioensayos que integran la exposición y los efectos de los contaminantes, que permiten representar sus interacciones con las diferentes propiedades físico-químicas y/o biológicas del suelo y evaluar los efectos de sus mezclas tanto en plantas como microorganismos (Chapman et al. 2012).

El presente trabajo tuvo como objetivo determinar la toxicidad de los metales pesados de $\mathrm{Cu}, \mathrm{Cd}, \mathrm{Ni}, \mathrm{Pb}$ y $\mathrm{Zn}$ en dos suelos agrícolas colombianos de los órdenes Andisol y Vertisol, mediante bioensayos con la planta indicadora Lactuca sativa y la bacteria Vibrio fischeri en extracto acuoso o solución del suelo, usando valores de referencia de dichos metales para suelos contaminados de Andalucía, España, con el fin de identificar su posible afectación a la biota del suelo y aportar a la estructuración de normatividad propia sobre la contaminación de suelos.

\section{MATERIALES Y MÉTODOS}

La investigación fue llevada a cabo en el Laboratorio de Edafología y Química Agrícola y en el Centro de Instrumentación Científica de la Universidad de Granada, España (Latitud: $37^{\circ} 10^{\prime} 41^{\prime \prime} \mathrm{N}$, Longitud: 336' 3'”O).

Se seleccionaron dos suelos agrícolas de Colombia, un Andisol Typic Melanudand tomado de la vereda La Aurelia, municipio de Cajibío (Latitud: $2^{\circ} 37^{\prime} 26^{\prime \prime} \mathrm{N}$, Longitud: $76^{\circ} 34^{\prime} 23^{\prime \prime} \mathrm{O}$ ), departamento del Cauca, y un Vertisol, Ustic Epiaquert en el Centro Experimental de la Universidad Nacional de Colombia, CEUNP, municipio de Candelaria (Latitud: $3^{\circ} 24^{\prime} 35^{\prime \prime} \mathrm{N}$, Longitud: $76^{\circ}$ $20^{\prime} 52^{\prime \prime}$ ), departamento del Valle del Cauca. Los suelos fueron muestreados a una profundidad de $0-20 \mathrm{~cm}$, secados al aire y tamizados con malla No. 10 para determinar densidad aparente (Da, método del cilindro), pH del suelo (suelo:agua relación1:2,5), textura (Loveland y Whalley 1991), contenido de humedad, mineralogía de arcilla (Martín 2004), contenido de carbono orgánico (CO) (IGAC 2006), bases intercambiables y capacidad de intercambio catiónico (CIC, acetato de amonio $1 \mathrm{~N} \mathrm{a} \mathrm{pH} \mathrm{7),} \mathrm{fósforo} \mathrm{(Bray} \mathrm{II),}$ contenido total de $\mathrm{Ni}, \mathrm{Cu}, \mathrm{Zn}, \mathrm{Cd}$ y $\mathrm{Pb}$ (digestión ácida; usando espectrofotómetro ICP-MS NEXION 300D) (US EPA 1999),y elementos mayores (Fluorescencia de Rayos $X$ en equipo Philips PW-1404) (Cortés et al. 2015).

Los suelos fueron contaminados de forma individual en el laboratorio con los metales $\mathrm{Ni}, \mathrm{Cu}, \mathrm{Zn}, \mathrm{Cd}$ y $\mathrm{Pb}$; las soluciones de cada uno de los metales se prepararon a partir de 
las siguientes sales: $\mathrm{Cd}\left(\mathrm{NO}_{3}\right)_{2}, \mathrm{ZnCl}_{2}, \mathrm{Ni}\left(\mathrm{NO}_{3}\right)_{2}$, $\mathrm{Cu}\left(\mathrm{NO}_{3}\right)_{3}$ y $\mathrm{Pb}\left(\mathrm{NO}_{3}\right)_{2}$, hasta superar los valores de referencia para el nivel de intervención de suelos agrícolas propuestos por la Junta de Andalucía: $\mathrm{Ni}\left(348 \mathrm{mg} \mathrm{kg}^{-1}\right), \mathrm{Cu}\left(626 \mathrm{mg} \mathrm{kg}^{-1}\right)$, Zn (1265 $\left.\mathrm{mg} \mathrm{kg}^{-1}\right), \mathrm{Cd}\left(27 \mathrm{mg} \mathrm{kg}^{-1}\right)$, y Pb $(660$ $\mathrm{mg} \mathrm{kg}{ }^{-1}$ ) (CMAJA 1999). Para ambos suelos se incluyeron muestras no contaminadas o control. Posteriormente, las muestras fueron incubadas bajo condiciones de invernadero, a una temperatura de $25^{\circ} \mathrm{C}$ y $60 \%$ de humedad del aire, en equipo GROW470/HR, simulando el ciclo diurno-nocturno de 12 horas, durante 60 y 90 días, y humedad gravimétrica del suelo del $60 \%$. Las unidades experimentales en vasos plásticos Eppendorf de $50 \mathrm{~cm}^{3}$ contenían las muestras de suelo tratadas (5 g cada una). Todos los tratamientos se realizaron por triplicado.

Se prepararon suspensiones de suelo: agua, relación 1:5 (peso: volumen), con las muestras de suelos contaminadas y no contaminadas, que fueron agitadas durante 12 horas; posteriormente se centrifugaron a 3000 r.p.m. El sobrenadante fue separado en dos porciones: la primera para la determinación de metales solubles en agua, en equipo ICP_MS NEXION 300D y la segunda para bioensayos de toxicidad, previa medición de $\mathrm{pH}$ y conductividad eléctrica (CE) (USDA 1998).

Para este estudio fueron seleccionadas dos pruebas de toxicidad:

a. Prueba de germinación de semillas/ elongación de raíz, de acuerdo a las recomendaciones de la US EPA (1996). Este test evaluó el efecto fitotóxico en la germinación de las semillas y el crecimiento inicial de las plántulas. En cajas Petri, fueron incubadas a $25 \pm 1^{\circ} \mathrm{C}$, 10 semillas de Lactuca sativa con 5 $\mathrm{ml}$ de extracto acuoso de suelos contaminados con metales y después de 5 días se midieron el número de semillas germinadas y la longitud de las raíces en desarrollo. El criterio de valoración fue la reducción calculada en la elongación de la raíz (LsR) en comparación con el control. b. Prueba de Microtox $®$ se basó en la reducción de la luminiscencia (ASTM 2004), a 5 min y 15 min, después de la mezcla de la bacteria marina Vibrio fischeri con los extractos acuosos. La prueba se realizó en un analizador de Microtox 500 (Microbics Corporation), de acuerdo con modificación para extractos acuosos (Martín et al. 2010). Los resultados se expresaron como la reducción de luminiscencia $(\mathrm{VfR})$ en comparación con el control.

Los valores del Índice de toxicidad (It) pueden variar desde $<0$ hasta 100 (máxima toxicidad), y con ellos se establecieron los siguientes cinco rangos de toxicidad (Escoto et al. 2007):

(a) $<0 \%$ toxicidad nula, indica la estimulación de la elongación radicular (mayor elongación en el tratamiento que en el agua destilada)

(b) 0 a $25 \%$ toxicidad baja,

(c) $>25$ a $50 \%$ toxicidad moderada,

(d) $>50$ a $75 \%$ toxicidad alta,

(e) $>75$ a $100 \%$ toxicidad muy alta.

La distribución normal de las variables fue establecida con la prueba de KolmogorovSmirnov. Las diferencias significativas se determinaron mediante ANOVA y las comparaciones múltiples con la prueba de Duncan $(p \leq 0,001)$. Se realizaron análisis de componentes principales con el fin de analizar la influencia de las propiedades del suelo en la capacidad de extracción y la toxicidad de los metales. Todos estos análisis se realizaron con un nivel de confianza del 95\% mediante el uso de SPSS v.20.0 (SPSS Inc. Chicago, USA).

\section{RESULTADOS Y DISCUSIÓN}

El Andisol es rico en arcillas amorfas (alófanos), textura franco limosa, $\mathrm{pH}$ moderadamente ácido como resultado del manejo con cal dolomita, altos niveles de $\mathrm{CO}, \mathrm{K}$ y $\mathrm{ClC}$, medios de $\mathrm{Ca}$ y $\mathrm{Mg}$, normal en $\mathrm{Na}$ y $\mathrm{C} / \mathrm{N}$, baja saturación de bases y bajo contenido de fósforo soluble. Buen contenido de agua útil, y baja densidad 
TEMAS AGRARIOS - Vol. 22:(2) Julio - Diciembre 2017 (43 - 53)

aparente. El Vertisol presenta textura franco arcillosa, presencia de arcillas esmectitas (tipo 2:1), pH neutro, altos niveles de Ca, Mg y de $\mathrm{P}$ disponible, normal en $\mathrm{Na}$ y $\mathrm{K}$, alta densidad aparente, buen contenido de agua útil, baja CIC y baja relación C/N (Cortés et al. 2015). Ambos suelos presentan concentraciones de metales pesados dentro del rango normal de suelos agrícolas (Kabata-Pendias 2011) (Tabla 1).

Tabla 1. Caracterización fisicoquímica de los suelos andisol (Cauca) y vertisol (Valle del Cauca).

\begin{tabular}{|c|c|c|c|c|c|c|c|c|c|c|c|}
\hline \multicolumn{6}{|c|}{ ANDISOL } & \multicolumn{6}{|c|}{ VERTISOL } \\
\hline \multicolumn{12}{|c|}{ PROPIEDADES FÍSISCAS } \\
\hline \multicolumn{2}{|c|}{ Humedad (\%) } & \multirow{2}{*}{$\begin{array}{c}\mathrm{Da} \\
\mathrm{g} \mathrm{cm}^{2}\end{array}$} & \multirow[t]{2}{*}{ Arenas } & \multirow{2}{*}{$\begin{array}{c}\text { Arcillas } \\
\% \\
\end{array}$} & \multirow[t]{2}{*}{ Limos } & \multicolumn{2}{|c|}{ Humedad (\%) } & \multirow{2}{*}{$\begin{array}{c}\mathrm{Da} \\
\mathrm{g} \mathrm{cm}^{2}\end{array}$} & \multirow[t]{2}{*}{ Arenas } & \multirow{2}{*}{$\begin{array}{c}\text { Arcillas } \\
\%\end{array}$} & \multirow[t]{2}{*}{ Limos } \\
\hline $\mathrm{CC}$ & PMP & & & & & $\mathrm{CC}$ & PMP & & & & \\
\hline 22,59 & 13,92 & 0,59 & 36,2 & 13,7 & 50,1 & 59,22 & 47,79 & 1,53 & 36,77 & 32,02 & 31,2 \\
\hline \multicolumn{12}{|c|}{ PROPIEDADES QUÍMICAS } \\
\hline $\begin{array}{c}\mathrm{pH} \\
1: 2,5 \\
\end{array}$ & $\mathrm{CO}$ & $\begin{array}{c}\mathrm{MO} \\
\%\end{array}$ & $\mathrm{~N}$ & $\begin{array}{l}\text { Psoluble } \\
\mathrm{mg} \mathrm{kg}^{-1} \\
\end{array}$ & Alófanos & $\begin{array}{c}\mathrm{pH} \\
1: 2,5 \\
\end{array}$ & $\mathrm{CO}$ & $\begin{array}{c}\mathrm{MO} \\
\%\end{array}$ & $\mathrm{~N}$ & $\begin{array}{l}\text { Psoluble } \\
\mathrm{mg} \mathrm{kg}^{-1} \\
\end{array}$ & Alófanos \\
\hline 5,56 & 9,7 & 16,72 & 0,87 & 2,34 & + & 7,15 & 0,71 & 1,22 & 0,53 & 41,54 & \\
\hline $\mathrm{ClC}$ & $\mathrm{Mg}^{2+}$ & $\mathrm{Ca}^{2+}$ & $\mathrm{Na}^{+}$ & $\mathrm{K}^{+}$ & SB & $\mathrm{CIC}$ & $\mathrm{Mg}^{2+}$ & $\mathrm{Ca}^{2+}$ & $\mathrm{Na}^{+}$ & $\mathrm{K}^{+}$ & SB \\
\hline \multicolumn{4}{|c|}{$\mathrm{Cmol}^{+} \mathrm{Kg}^{-1}$} & & $\%$ & \multicolumn{4}{|c|}{$\mathrm{Cmol}^{+} \mathrm{Kg}^{-1}$} & & $\%$ \\
\hline 54,98 & 1,53 & 5,36 & 0,05 & 0,36 & 13,3 & 21,2 & 6,59 & 11,16 & 0,25 & 0,29 & 86,3 \\
\hline $\mathrm{Ni}$ & $\mathrm{Cu}$ & $\mathrm{Zn}$ & $\mathrm{Cd}$ & \multirow{2}{*}{\multicolumn{2}{|c|}{$\mathrm{Pb}$}} & $\mathrm{Ni}$ & $\mathrm{Cu}$ & $\mathrm{Zn}$ & $\mathrm{Cd}$ & \multirow{2}{*}{\multicolumn{2}{|c|}{$\mathrm{Pb}$}} \\
\hline \multicolumn{4}{|c|}{$\mathrm{mg} \mathrm{kg}^{-1}$} & & & \multicolumn{4}{|c|}{$\mathrm{mg} \mathrm{kg}^{-1}$} & & \\
\hline 8,76 & 45,28 & 92,63 & 0,35 & 20, &, 41 & 19,1 & 42,86 & 73,89 & 0,68 & \multicolumn{2}{|c|}{15,31} \\
\hline
\end{tabular}

Los extractos acuosos del Andisol se clasifican como ligeramente ácidos, en tanto que los del Vertisol se ubican dentro del rango considerado como de neutralidad de las aguas naturales
(Tabla 2); así mismo, su conductividad eléctrica es alta e implica posibilidad de afectación de aguas superficiales y subterráneas.

Tabla 2. Caracterización de los extractos acuosos de un suelo andisol y un vertisol ( $\left.\mathrm{mg} \mathrm{l}^{-1}\right)$.

\begin{tabular}{|c|c|c|c|c|c|c|c|c|}
\hline \multirow[b]{2}{*}{ Suelo } & \multirow[b]{2}{*}{ Tratamiento } & \multirow[b]{2}{*}{ Metal } & \multicolumn{3}{|c|}{ Periodo 1} & \multicolumn{3}{|c|}{ Periodo 2} \\
\hline & & & $\mathrm{pH}$ & CE & $\begin{array}{c}\text { Concentración } \\
\text { mg }\left.\right|_{-1} ^{-1}\end{array}$ & $\mathrm{pH}$ & CE & $\begin{array}{c}\text { Concentración } \\
\left.\mathrm{mg}\right|^{-1}\end{array}$ \\
\hline \multirow{10}{*}{ Andisol } & \multirow{5}{*}{ SNC } & CuW & & & $\mathrm{Bdl}$ & & & 0,023 \\
\hline & & $\mathrm{ZnW}$ & & & 0,006 & & & 0,122 \\
\hline & & $\mathrm{NiW}$ & 5,56 & 0,45 & 0,012 & 5,54 & 0,45 & 0,025 \\
\hline & & $\mathrm{PbW}$ & & & 0,002 & & & 0,002 \\
\hline & & $\mathrm{CdW}$ & & & Bdl & & & 0,020 \\
\hline & \multirow{5}{*}{ SC } & CuW & 4,91 & 2,10 & 0,149 & 5,10 & 2,30 & 0,151 \\
\hline & & $\mathrm{ZnW}$ & 4,79 & 4,30 & 21,737 & 4,93 & 3,55 & 18,104 \\
\hline & & $\mathrm{NiW}$ & 5,14 & 1,50 & 1,292 & 5,50 & 1,70 & 1,086 \\
\hline & & $\mathrm{PbW}$ & 5,28 & 1,15 & 0,163 & 5,60 & 1,25 & 0,139 \\
\hline & & CdW & 5,93 & 0,50 & 0,009 & 5,87 & 0,85 & 0,007 \\
\hline \multirow{10}{*}{ Vertisol } & \multirow{5}{*}{$\mathrm{SNC}$} & CuW & & & 0,026 & & & 0,016 \\
\hline & & $\mathrm{ZnW}$ & & & 0,038 & & & 0,010 \\
\hline & & $\mathrm{NiW}$ & 7,82 & 0,45 & 0,017 & 7,87 & 0,65 & 0,004 \\
\hline & & $\mathrm{PbW}$ & & & 0,008 & & & 0,001 \\
\hline & & $\mathrm{CdW}$ & & & Bdl & & & Bdl \\
\hline & \multirow{5}{*}{ SC } & CuW & 6,87 & 2,35 & 0,185 & 7,03 & 2,20 & 0,321 \\
\hline & & $\mathrm{ZnW}$ & 6,54 & 4,20 & 6,160 & 6,76 & 3,75 & 4,572 \\
\hline & & NiW & 7,17 & 2,00 & 0,859 & 7,34 & 1,60 & 0,546 \\
\hline & & PbW & 7,30 & 1,05 & 0,305 & 7,53 & 1,20 & 0,319 \\
\hline & & $\mathrm{CdW}$ & 7,45 & 0,50 & 0,004 & 7,60 & 0,65 & Bdl \\
\hline
\end{tabular}

SNC: Suelo no contaminado; SC: suelo contaminado; $\mathrm{CE}$ : Conductividad eléctrica $(\mathrm{mS} / \mathrm{cm})$; bdl = por debajo del límite de detección; XW: Concentración de metal extraído en agua. 
Las concentraciones solubles de los metales en los extractos superan los valores críticos de toxicidad para la solución del suelo (Bohn 1993), por lo que se les considera con alta capacidad de acumulación en los organismos y fuerte toxicidad (Ayers y Westcot 1985; Real Decreto 927/1988; Real Decreto 140/2003; OMS 2006). El mayor contenido de PbW en el extracto del vertisol en relación con el del andisol, puede deberse al bajo contenido de óxidos de hierro y manganeso que posee este tipo de suelo, con los cuales este metal tiene alta afinidad (Cerqueira et al. 2011).

La menor concentración de ZnW en el vertisol está relacionada con el alto contenido de fósforo soluble, pues afecta el potencial eléctrico de los coloides haciéndolo más negativo (principalmente por los óxidos de hierro) y facilita la adsorción del metal (PérezNovo et al. 2011).
La germinación de lechugas en el control (agua destilada) fue del $97 \%$ y $100 \%$ en los periodos de incubación 1 y 2, respectivamente, por lo que se considera que la prueba es fiable (OECD 2006). No existen diferencias significativas ( $p>0,05)$ entre los distintos tratamientos, ni con respecto al control, infiriéndose que los metales en la solución del suelo no inhiben la germinación de la Lactuca sativa (no hay toxicidad).

De acuerdo al test de Duncan $(p<0,001)$ (Figura 1) se encontró:

-Tratamientos sin efecto fitotóxico. En estos tratamientos, las elongaciones no difieren de las del blanco (suelo no contaminado) ni del control (agua destilada). Para los extractos del Andisol, periodo 1, los tratamientos son: $\mathrm{Pb}$, $\mathrm{Cu}$ y $\mathrm{Cd}$. Se produce hormesis (un fenómeno de relación entre la dosis y la respuesta, caracterizada por estimulación a bajas dosis e inhibición con altas dosis) para el Cd. En el

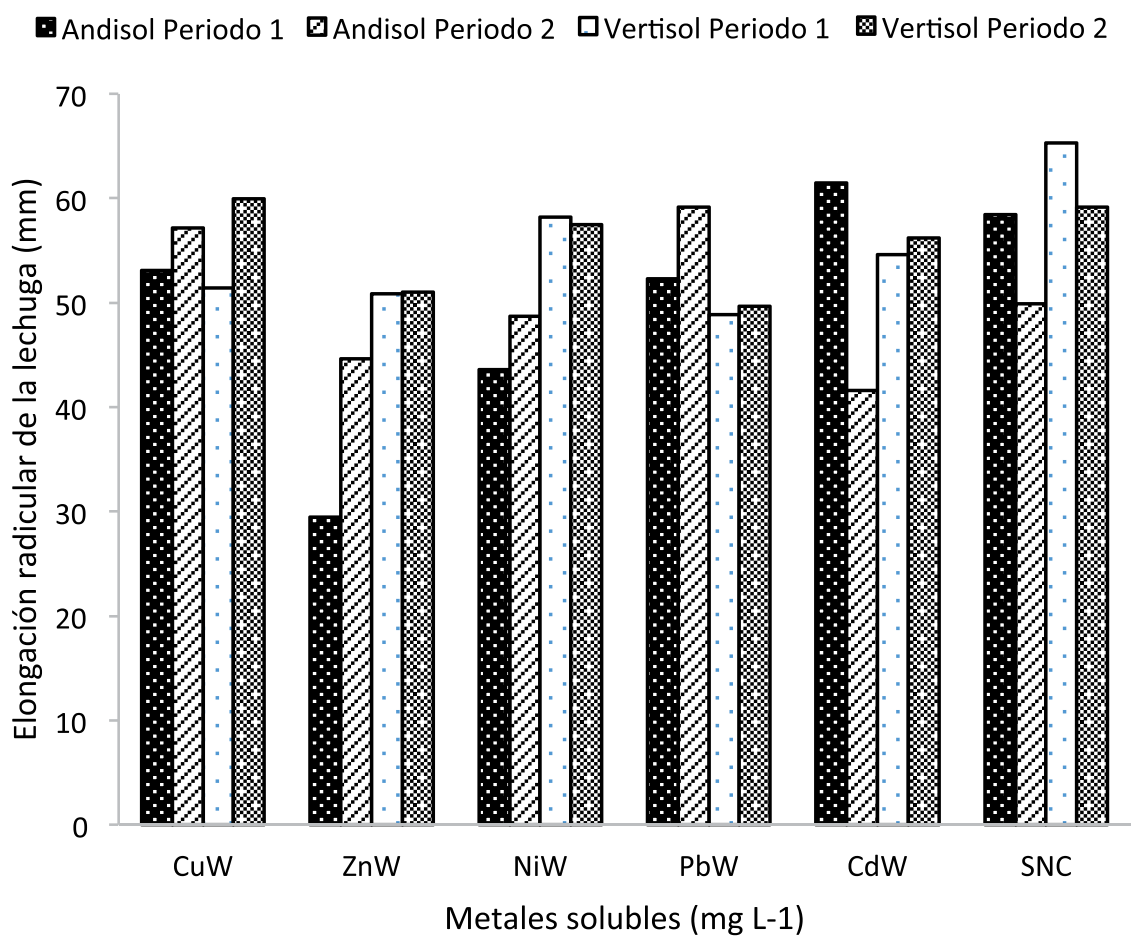

Figura 1. Elongación radicular de la lechuga $(\mathrm{mm})$ en los diferentes tratamientos. SNC: Suelo no contaminado; S.D: Desviación estándar; *: Diferencias significativas en relación con el SNC $(\mathrm{p}<0,05)$ 
periodo 2, los tratamientos son: $\mathrm{Pb}, \mathrm{Cu}, \mathrm{Ni}$ y $\mathrm{Zn}$. Se presenta hormesis para el Pb. Para los extractos del vertisol, en los dos periodos de incubación, los tratamientos son: $\mathrm{Cu}, \mathrm{Cd}, \mathrm{Pb}$, Ni y Zn. En el periodo 1, la muestra de suelo no contaminado (blanco) presenta hormesis.

-Tratamientos con efecto fitotóxico. Estos efectos fitotóxicos sólo se presentan para los extractos del andisol, en el periodo 1, para $\mathrm{Ni} \mathrm{y}$ Zn; y en el periodo 2, para el $\mathrm{Cd}$.

La toxicidad en la $L$. sativa generada en el tratamiento ZnW (Tabla 3), se presentó de manera moderada en el Andisol, periodo 1, debido posiblemente a que la lechuga es sensible al pH ácido de la solución de dicho tratamiento, lo cual inhibe su crecimiento y metabolismo celular (Chapman et al. 2012).

El incremento de la toxicidad para el $\mathrm{Cd}$ en el andisol, periodo 2 de incubación, en el cual la concentración es menor, lo cual puede atribuirse a una posible desnitrificación (Cornu et al. 2008), así como a un cambio del potencial redox, al aumento de la conductividad eléctrica de la solución, y que por aumento de la acidez, este metal pudo generar el desplazamiento de las sales de los sitios de intercambio de los coloides del extracto acuoso, generando la inhibición del crecimiento de las plántulas (Rodríguez-Serrano et al. 2008).

El vertisol presentó la mayor capacidad de inhibición de la toxicidad de los metales pesados sobre la Lactuca sativa, debido posiblemente a que a mayor valor del $\mathrm{pH}$ se disminuye la movilidad y biodisponibilidad de los metales, así como al mayor contenido de arcillas, cuya gran superficie específica le confiere gran capacidad de adsorción de metales (Kabata-Pendias 2011).

El pH y la conductividad eléctrica de los extractos de los suelos están dentro del rango recomendado para realizar el bioensayo con Vibrio fischeri (Onorati y Mecozzi 2004). El Test de Duncan $(p<0,001)$ aplicado a prueba de toxicidad por luminiscencia, a 5 y 15 minutos, para los dos periodos de incubación (Tabla 4), muestra que los tratamientos se diferencian en 2 grupos:

Tabla 3. Toxicidad en Lactuca sativa y Vibrio fischeri en un suelo andisol y un vertisol.

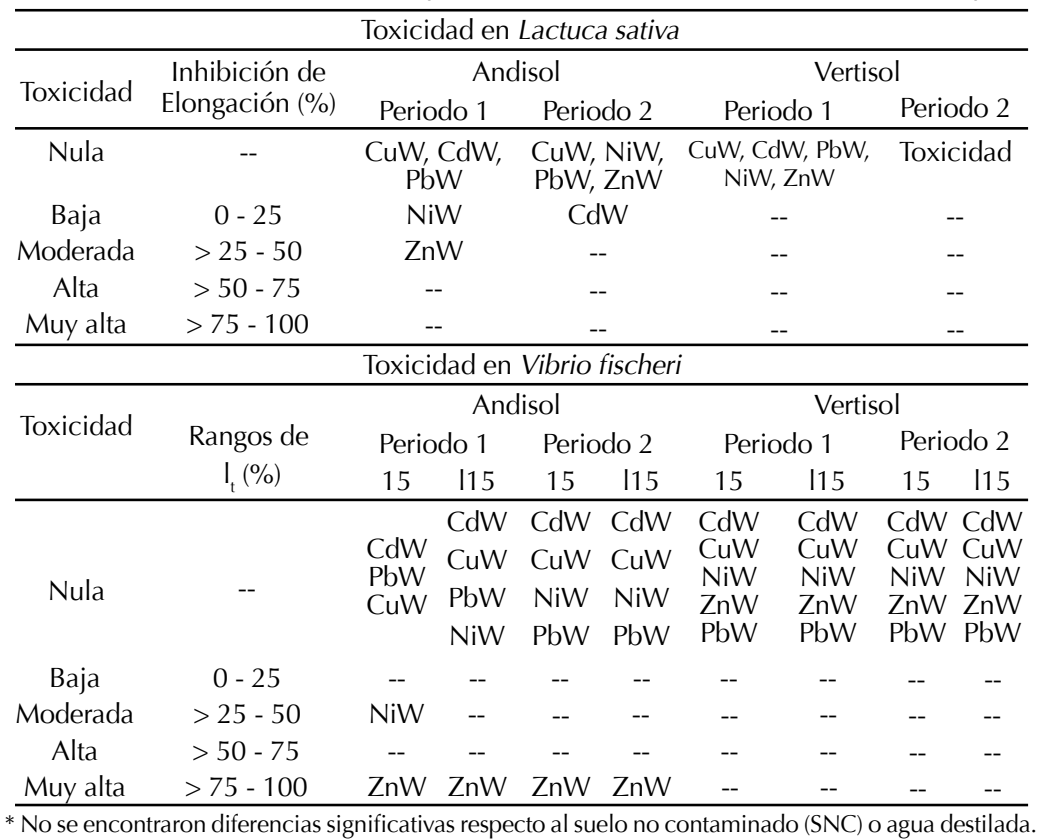


Cortés et al. - Toxicidad por metales en suelos agrícolas

Tabla 4. Reducción de la luminiscencia en Vibrio fischeri (\%), de un suelo andisol y un vertisol

\begin{tabular}{|c|c|c|c|c|c|c|c|c|}
\hline \multirow{3}{*}{ Metal } & \multicolumn{4}{|c|}{$\begin{array}{c}\text { Andisol } \\
\mathrm{t}=5 \text { minutos }\end{array}$} & \multicolumn{4}{|c|}{$\begin{array}{c}\text { Vertisol } \\
\mathrm{t}=5 \text { minutos }\end{array}$} \\
\hline & \multicolumn{2}{|c|}{ Incubació 1} & \multicolumn{2}{|c|}{ Incubació 2} & \multicolumn{2}{|c|}{ Incubació 1} & \multicolumn{2}{|c|}{ Incubació 2} \\
\hline & Promedio & SD & Promedio & SD & Promedio & SD & Promedio & $\mathrm{SD}$ \\
\hline CuW & 56,04 & 1,89 & 38,84 & 5,51 & 32,76 & 3,70 & 42,48 & 10,17 \\
\hline $\mathrm{ZnW}$ & 96,49 & 1,44 & 96,45 & 2,85 & 62,34 & 8,21 & 61,79 & 0,53 \\
\hline $\mathrm{NiW}$ & 65,88 & 8,69 & 39,55 & 6,64 & 73,45 & 5,40 & 42,44 & 1,42 \\
\hline PbW & 57,58 & 7,09 & 65,22 & 7,93 & 56,71 & 7,81 & 50,69 & 1,83 \\
\hline CdW & 47,12 & 4,02 & 38,04 & 1,33 & 62,16 & 1,71 & 56,67 & 5,13 \\
\hline \multirow[t]{3}{*}{ SNC } & 45,73 & 3,71 & 66,74 & 9,49 & 69,00 & 9,66 & 60,88 & 5,82 \\
\hline & \multicolumn{4}{|c|}{$t=5$ minutos } & \multicolumn{4}{|c|}{$t=5$ minutos } \\
\hline & Promedio & SD & Promedio & SD & Promedio & SD & Promedio & SD \\
\hline CuW & 56,11 & 2,37 & 47,67 & 4,25 & 38,05 & 10,17 & 49,40 & 12,57 \\
\hline ZnW & $98,95 *$ & 0,25 & $98,65^{*}$ & 0,63 & $81,88^{*}$ & 6,50 & $85,54 *$ & 1,85 \\
\hline $\mathrm{NiW}$ & 68,68 & 11,17 & 46,95 & 6,03 & 67,00 & 4,20 & 46,97 & 0,70 \\
\hline PbW & 61,09 & 3,91 & 73,33 & 6,75 & 59,88 & 2,88 & 61,34 & 2,04 \\
\hline $\mathrm{CdW}$ & 52,39 & 1,64 & 45,39 & 2,06 & 64,75 & 1,98 & 66,78 & 4,87 \\
\hline SNC & 55,79 & 4,95 & 76,44 & 7,69 & 73,98 & 8,41 & 73,52 & 8,81 \\
\hline
\end{tabular}

Tratamientos sin efecto fitotóxico. En estos tratamientos los valores de luminiscencia no difieren de las del blanco (suelo no contaminado) ni del control (agua destilada). Para el Andisol, en el periodo de incubación 1, a 5 y 15 minutos: $\mathrm{Cd}, \mathrm{Cu}, \mathrm{Pb}$; a 15 minutos, además está el $\mathrm{Ni}$, y para $\mathrm{Cd}$ y $\mathrm{Cu}$ se produce hormesis. En el periodo 2, a 5 y 15 minutos: $\mathrm{Cd}, \mathrm{Cu}, \mathrm{Ni}$ y $\mathrm{Pb}$. Para $\mathrm{Cd}, \mathrm{Cu}, \mathrm{Ni}$ se presenta hormesis. Para el vertisol, en el periodo de incubación 1, a 5 y 15 minutos: $\mathrm{Cd}, \mathrm{Cu}, \mathrm{Ni}, \mathrm{Pb}$ y $\mathrm{Zn}$; en el periodo 2, a 5 y 15 minutos: $\mathrm{Cd}, \mathrm{Cu}$, $\mathrm{Ni}, \mathrm{Pb}$ y $\mathrm{Zn}$.

Tratamientos con efecto fitotóxico. El efecto fitotóxico sólo se presentan para el tratamiento con Zn, en el andisol, en ambos periodos de incubación, y para los dos momentos de medida.

De los índices de toxicidad para Vibrio fischeri (Tabla 3) se infiere que la capacidad de inhibición de la toxicidad de ambos suelos es alta, en virtud a que sus concentraciones son muy tóxicas para la solución del suelo (Bohn 1993) y para la bacteria (Hsieh et al. 2004).

El vertisol presentó la mayor capacidad de inhibición de la toxicidad, debido posiblemente al mayor valor del $\mathrm{pH}$, al mayor contenido y tipo de arcillas.

El metal que influyó significativamente en la inhibición de la luminiscencia fue el $\mathrm{Zn}$, que por su concentración, bajo radio iónico, naturaleza bactericida y antimicrobiana, pudo afectar fuertemente los sistemas enzimáticos de la bacteria (Fulladosa et al. 2005). No obstante, en ambos suelos se obtuvo nula toxicidad hacia la bacteria por parte de CdW, CuW, PbW, así como el NiW en el vertisol, debido a que las paredes celulares bacterianas funcionan como polielectrólitos, los cuales adsorben los iones metálicos de la solución mediante atracción electrostática, complementada con fuerzas de Van der Waals, enlaces covalentes, interacciones redox y precipitación celular (Huang et al. 2008).

El análisis de componentes principales (Figura 2) explica el $96,19 \%$ de la varianza; el $55,65 \%$ corresponde al componente 1 , y el $40,54 \%$ al componente 2. En éste se observa que los parámetros de mayor influencia sobre la toxicidad de los organismos en ensayo (LsR, VfR5 y VfR15) son las concentraciones de los metales solubles y la conductividad eléctrica 


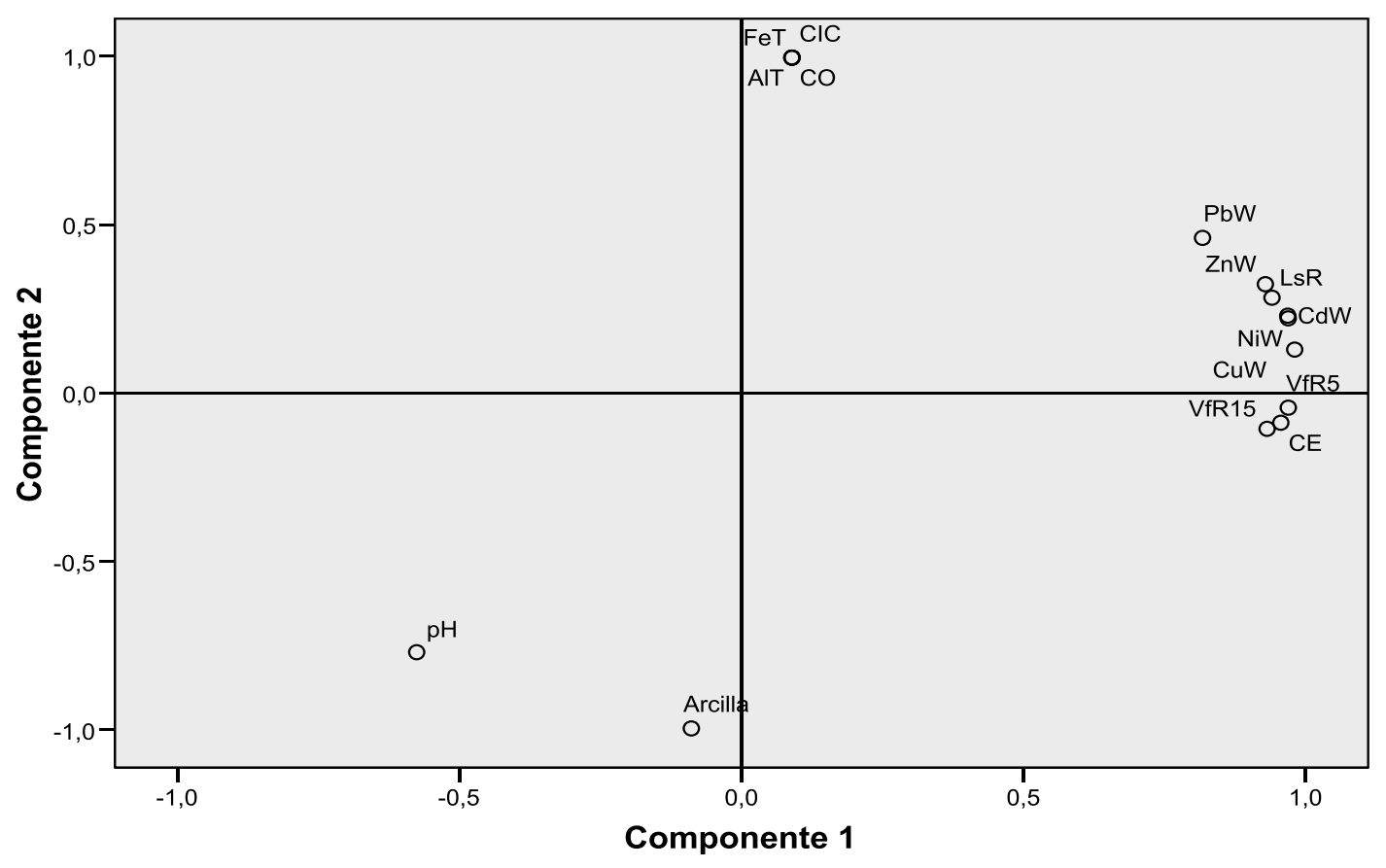

Figura 2. Análisis de componentes principales entre propiedades de un suelo andisol y un vertisol.

del extracto del suelo, lo cual indica que la concentración de los metales usada en estos ensayos afectan significativamente el desarrollo de la biota del suelo; por otra parte, la capacidad de intercambio catiónico se asocia al Fe, Al y $\mathrm{CO}$ y el $\mathrm{pH}$ al contenido de arcilla.

Los niveles de intervención para suelos basados en concentraciones totales de metales no representan criterio suficiente para declarar un suelo como contaminado debido a que su movilidad depende de las propiedades de ellos, siendo importante el realizar pruebas de biodisponibilidad, bioensayos de toxicidad y de abundancia, diversidad, tolerancia y capacidad de remediación de la contaminación por parte de los organismos edáficos (Cortés et al. 2015; Sarria et al. 2015).

\section{CONCLUSIONES}

La capacidad de inhibición de la toxicidad de los suelos (andisol y vertisol) sobre la planta indicadora Lactuca sativa y la bacteria Vibrio fischeri es alta, dado que las concentraciones solubles de los metales se consideran toxicas en la solución del suelo; , dicha capacidad se incrementó con el tiempo, y se relacionó con la reducción de las concentraciones solubles de metales como resultado del incremento del $\mathrm{pH}$ que permitió una mayor fijación en las posiciones de adsorción del suelo (materia orgánica, arcillas, y complejos amorfos de Fe y Al).

El Vertisol presentó una mayor capacidad de inmovilización de los metales pesados, reflejándose en una menor toxicidad sobre los organismos del bioensayo y actuando como un factor de protección contra la movilidad de los contaminantes en el suelo. Las propiedades del suelo que parecen haber sido más efectivas en dicha inmovilización fueron la arcilla y el $\mathrm{pH}$.

El Zn fue el metal que influyó significativamente sobre la inhibición de la luminiscencia de la bacteria Vibrio fischeri, posiblemente por su mayor concentración, pH ácido y mayor movilización. 
La bacteria bioluminiscente Vibrio fischeri mostró una mayor sensibilidad a todos los metales pesados evaluados, permitiendo una mejor discriminación de niveles de toxicidad. Más sin embargo, la interacción bacteria-metal es importante para deducir adecuadamente la toxicidad generada, ya que éstas poseen mecanismos de tolerancia ante la contaminación.

\section{AGRADECIMIENTOS}

Ismael Vivas por proveernos los suelos del municipio de Cajibío, departamento del Cauca, al Departamento de Edafología de la Universidad de Granada, al Grupo de Investigación en Uso y Manejo de Suelos y Aguas de la Universidad Nacional de Colombia.

\section{REFERENCIAS}

ASTM (American Society for Testing and Materials). 2004. Standard Test Method for Assessing the Microbial Detoxification of Chemically Contaminated Water and Soil Using a Toxicity Test with a Luminescent Marine Bacterium. ASTM D5660 - 96. USA.

Ayers, R. y Westcot, D. 1985. Water quality for agriculture. FAO. Roma, Italia. http:// www.fao.org/docrep/003/T0234E/ T0234E00.htm\#pre

Bohn, X. (1 raEd.). 1993. Química del suelo, México D.F, México: Editorial Limusa, S.A., de C.V.

Bolan, N., Kunhikrishnan, A., Thangarajan, R., Kumpiene, J., Park, J., Makino, T., Kirkham, M. and Scheckel, K. 2014. Review: Remediation of heavy metal(loid) s contaminated soils - To mobilize or to immobilize?. Journal of Hazardous Materials 266: 141-166. https://doi. org/10.1016/j.jhazmat.2013.12.018.
Cerqueira, B., Covelo, E., Andrade, M. y Vega, F. 2011. Retention and Mobility of Copper and Lead in Soils as Influenced by Soil Horizon Properties. Pedosphere 21 (5): 603-614. https://doi.org/10.1016/ S1002-0160(11)60162-8.

Chapman, E., Helmer, S., Dave, G. and Murimboh, J. 2012. Utility of bioassays (lettuce, red clover, red fescue, Microtox, MetSTICK, Hyalella, bait lamina) in ecological risk screening of acid metal (Zn) contaminated soil. Ecotoxicology and Environmental Safety 80:161-171. https:// doi.org/10.1016/j.ecoenv.2012.02.025.

CMAJA (Consejería del Medio Ambiente de la Junta de Andalucía). 1999. LoS criterios y estándares para declarar un suelo contaminado en Andalucía y la metodología y técnicas de toma de muestra y análisis para su investigación.

Cornu, J., Denaix, L., Schneider, A. and Pellerin, S. 2008. Temporal variability of solution $\mathrm{Cd}^{2+}$ concentration in metalcontaminated soils as affected by soil temperature: consequences on lettuce (Lactuca sativa L.) exposure. Plant Soil 307:51-65. https://doi.org/10.1007/ s11104-008-9580-x

Cortés, L., Bravo, I., Martin, F. y Menjivar, J. 2015. Adsorción de metales pesados en andisoles, vertisoles y ácidos húmicos. Acta Agronómica 64 (1): 61-71. https:// doi.org/10.15446/acag.v64n1.43677.

Cortés, L., Bravo, I., Martin, F. y Menjivar, J. 2016. Extracción secuencial de metales pesados en dos suelos contaminados (Andisol y Vertisol) enmendados con ácidos húmicos. Acta Agronómica 65 (3): 232-238. https://doi.org/10.15446/acag. v65n3.44485. 
Escoto, V., Fernández, G. y Martín, P. 2007. Determination of phytotoxicity of soluble elements in soils, based on a bioassay with lettuce (Lactuca sativa L.). Science of the Total Environment 378: 63-66. https:// doi.org/10.1016/j.scitotenv.2007.01.007.

Fulladosa, E., Murat, J., Martínez, M. y Villaescusa, I. 2005. Patterns of metals and arsenic poisoning in Vibrio fischeri bacteria. Chemosphere 60: 43-48. https://doi.org/10.1016/j. chemosphere.2004.12.026.

Hsieh, Chi-Ying., Tsai, Meng-Hsiun., Ryan, D. and Pancorbo, O. 2004. Toxicity of the 13 priority pollutant metals to Vibrio fischeri in the Microtox $\AA$ chronic toxicity test. The Science of the Total Environment 320: 37-50. https://doi.org/10.1016/ S0048-9697(03)00451-0.

Hu, Q., Qi, H., Zeng, J. and Zhang, H. 2007. Bacterial diversity in soils around a lead and zinc mine. Journal of Environmental Sciences 19: 74-79. https://doi. org/10.1016/S1001-0742(07)60012-6.

Huang, Q., Huang, P. and Violante, A. 2008. Soil Mineral- Microbe-Organic Interactions. Theories and Applications. SpringerVerlag Berlin Heidelberg. https://doi. org/10.1007/978-3-540-77686-4

Huang, Y., Li, T., Wu, C., He, Z., Japenga, J., Deng, M. and Yang, X. 2015. An integrated approach to assess heavy metal source apportionment in peri-urban agricultural soils. Journal of Hazardous Materials 299: 540-549. https://doi.org/10.1016/j. jhazmat.2015.07.041.

IGAC (Instituto Geográfico Agustín Codazzi). 2006. Métodos Analíticos de Laboratorio de Suelos. Imprenta Nacional de Colombia, Sexta Edición.

Kabata-Pendias, A. (4a Ed.). 2011. Trace Elements in Soils and Plants. Boca Raton, FL. United States, CRC Press.
Loveland, P. and Whalley, W. 1991. Particle size analysis. Soil Analysis: Physical Methods, New York, United States, Smith, K.A., Mullis, Ch.E. (Eds.), Marcel Dekker, pp. 271-328.

Marrugo-Negrete, J., Pinedo-Hernández, J. and Díez, S. 2017. Assessment of heavy metal pollution, spatial distribution and origin in agricultural soils along the Sinú River Basin, Colombia. Environmental Research 154: 380-388. https://doi. org/10.1016/j.envres.2017.01.021.

Martín, J. 2004. Xpowder. A software package for powder X-ray diffraction analysis.

Martín, F., Escoto, M., Fernández, J., Fernández, E., Arco, E., Sierra, M. and Dorronsoro, C. 2010. Toxicity assessment of sediments with natural anomalous concentrations in heavy metals by the use of bioassay. Int. J. Chem. Eng., 101390, 1-6. https://doi. org/10.1155/2010/101390.

OECD (Organisation for Economic Cooperation and Development). 2006. Terrestrial Plant Test $N^{\circ}$ 208: Seedling Emergence and Seedling Growth Test. Paris, France.

OMS (Organización Mundial de la Salud). 2006. Guías para la calidad del agua potable. 3era Ed. (1).

Onorati, F. and Mecozzi, M. 2004. "Effects of two diluents in the Microtox_toxicity bioassay with marine sediments," Chemosphere 54 (5): 679-687. https://doi.org/10.1016/j. chemosphere.2003.09.010

Pérez-Novo, C., Bermúdez-Couso, A., LópezPeriago, E., Fernández-Calviño, D. and Arias-Estévez, M. 2011. Zinc adsorption in acid soils Influence of phosphate. Geoderma 162: 358-364. https://doi. org/10.1016/j.geoderma.2011.03.008. 
Real Decreto 927/1988. de 29 de julio, por el que se aprueba el Reglamento de la Administración Pública del agua y de la planificación hidrológica, en desarrollo de los Títulos II y III de la Ley de Agua. BOE No 209 de 31 de Agosto de 1988.

Real Decreto 140/2003. de 7 de febrero, por el que se establecen los criterios sanitarios de la calidad del agua de consumo humano. BOE No 45, 21 febrero 2003.

Rodríguez-Serrano, M., Martínez-De La Casa, N., Romero-Puertas, M. C., Del Río, L.A. y Sandalio, L. 2008. Toxicidad del Cadmio en Plantas. Ecosistemas 17 (3): 139-146.

Sarria, M., Cortés, L. y Martín, F. 2015. Evaluación de la recuperación de suelos contaminados por el vertido de Aznalcóllar. Acta Agronómica 64 (2): 156-164. https://doi.org/10.15446/acag. v64n2.44265.
Sherameti, I. and Varma, A. 2010. Soil Biology: Soil Heavy Metals. Volume 19. SpringerVerlag Berlin Heidelberg.

US EPA (Environmental Protection Agency). 1996. Ecological effects test guidelines, in: Seed Germination/Root Elongation Toxicity Test, US Environmental Protection Agency, Office of Prevention, Pesticide and Toxic Substances (OPPTS) 850.4200, Washington DC, EPA 712-C-96-154.

\section{US EPA (Environmental Protection Agency).} 1999. Method 3051: microwave assisted acid digestion of sediments, sludges, soils and oils.

Wang, Q., Xie, Z. and Li, F. 2015. Using ensemble models to identify and apportion heavy metal pollution sources in agricultural soils on a local scale. Environmental Pollution 206: 227-235. 